\title{
Mathematical Characterization of the Tensile Deformation Curve of Cast Iron Materials
}

\author{
Ingvar L Svensson ${ }^{1, \mathrm{a}^{*}}$ and Kent Salomonsson ${ }^{1, \mathrm{~b}}$ \\ ${ }^{1}$ Jönköping University, School of Engineering \\ ${ }^{a}$ Materials and Manufacturing - Casting \\ ${ }^{b}$ Product Development -Simulation and Optimization \\ Box 1026, SE-553 13 Jönköping, Sweden \\ a ingvar.svensson@ju.se, ${ }^{b}$ kent.salomonsson@ju.se
}

Keywords: Tensile test curve, microstructure dependent deformation, mathematical curve fitting, evaluation of, curve fitting methods, microstructure dependent prediction of tensile test curve

\begin{abstract}
The manufacturing process gives cast iron castings properties which are dependent on component design, metallurgy and casting method. Factors such as local wall thickness influences the coarseness and type of microstructure and the castings will have local properties depending on the local metallurgical and thermal history. The stress/strain behaviour of cast materials is typically determined by performing a tensile test in a tensile test machine.

The deformation behaviour will normally be determined by two mechanisms, namely, elastic and plastic phenomena. The plastic behaviour is based on dislocation movements in the lattice. Commonly, the deformation history of cast iron involves elastic, plastic and crack phases. The cast iron material has a complex microstructure and first order equations cannot be used to predict the deformation during loading. Until methods have been developed, the characterization of complex microstructure materials such as cast iron has to be determined by use of empirical methods. The empirical methods have to couple the internal microstructure and composition of the material with deformation phenomena during loading.

The paper will show a method to characterize tensile test curves of cast iron materials which can be used to couple deformation phenomena with for example microstructure. The equations are aimed to make the tensile test curve ready for curve fitting and optimization in two steps.

Each stress/strain curve is like a finger print of the material and requires well performed tests and some advices are given.

The paper also wants to encourage researchers and people working with tensile testing to get out more of their effort to measure strength of cast iron materials and connect the result to the microstructure of the specimens.
\end{abstract}

\section{Introduction}

Simulation and prediction of properties of a material if often wanted and especially on materials which the properties are depending on the primary manufacturing process [1]. The properties of cast products are primarily determined by the casting process or after following heat treatment. The process parameters highly influence the micro structure and the following properties. The properties can be mechanical properties like tensile strength, elongation and yield strength and further more like hardness, fatigue properties and creep. Other physical properties like thermal conductivity, dilation and volume changes at heating and heat capacity are properties which are depending on the micro structure and chemical composition.

Tensile testing is a commonly used method in industry and in research to characterise cast materials. The tensile test curve reflects the behaviour of the interior of the material. The tensile test curve shows the influence of load versus elongation. A better understanding can be obtained if we could explore the responses of tensile and compression loads more.

The microstructure in a casting is normally very complex and cannot be easily described [2-6]. At present it is not possible to simulate a casted multiphase material to predict the mechanical 
properties in a generic way. Another approach is to analyse the tensile test curve a little more carefully. The curve is like a finger print that is unique to the castings microstructure and its chemical composition. Well performed instrumented measurements must be made to be able to evaluate the shape of the curve.

\section{Setup of a Tensile Strain - Stress Experiment}

A number of important points must be taken in to consideration in order to obtain a scientifically sound and reliable tensile test curve. Several international standards on how to perform tensile tests have been developed, for example ISO 6892. Another basic description can be found in J.R. Davis book on tensile testing [7] as well as in instructions by manufacturers of tensile test machines.

Here are some key points to consider when performing tensile testing of cast iron specimens;

- Well calibrated load cells and the grips in linear position. Using clip-on extensometer or even better, a contactless laser extensometer. Use the clip-on for the whole test cycle. Note that there is a risk to damage the clip-on extensometer.

- The specimens should be manufactured with no or very little introduced hardworking zones on the surface. Do not use high turning speeds for round samples. Use water or laser cutting for rectangular cross-section specimens.

- Use standardized shapes of specimens to relate to earlier work.

- Be sure of no sliding of the specimen in the tensile test machine grips.

- Control the rate of loading (e.g. $<30 \mathrm{MPa} / \mathrm{min}$ ) in the elastic area specially for SGI castings. Use same deformation speed during the whole test. Commonly, the deformation behaviour is strain rate dependent (Range $10^{-5-} 10^{-1} 1 / \mathrm{s}$ and normally in the range or $2-510^{-4} 1 / \mathrm{s}$ ).

- If possible, the number of test specimens should be three or more with the same material.

Independently of what value selected it is important to be consequent and in publications and test protocols state under which condition the testing were made.

\section{Characterization and Modelling of Microstructure Based Properties}

For different cast iron the elastic module is dependent on the graphite morphology shape and content. Measuring the elastic module is not easy in a tensile test machine due to small elongations. For grey iron, the elastic module is often presented at a certain load e.g. $50 \mathrm{MPa}$. Moreover, sometimes the elastic module is calculated by extrapolation to $0 \mathrm{MPa}$. Magnetic resonance vibration yields elasticity modulus close to zero load.

For cast iron, the load interval at which one calculates the tangent elastic module close to zero load varies depending on the type of cast iron. For lamellae graphite iron (LGI), the change is more apparent than for nodular cast iron (SGI), which show has little larger interval with constant elastic module, see Fig.'s 1a and 1c. Differentiation of the tensile test curves yields the results shown in Fig.'s 1a-1c. The elastic module at a certain stress level is called the tangent module, which is the tangent of the tensile test curve.
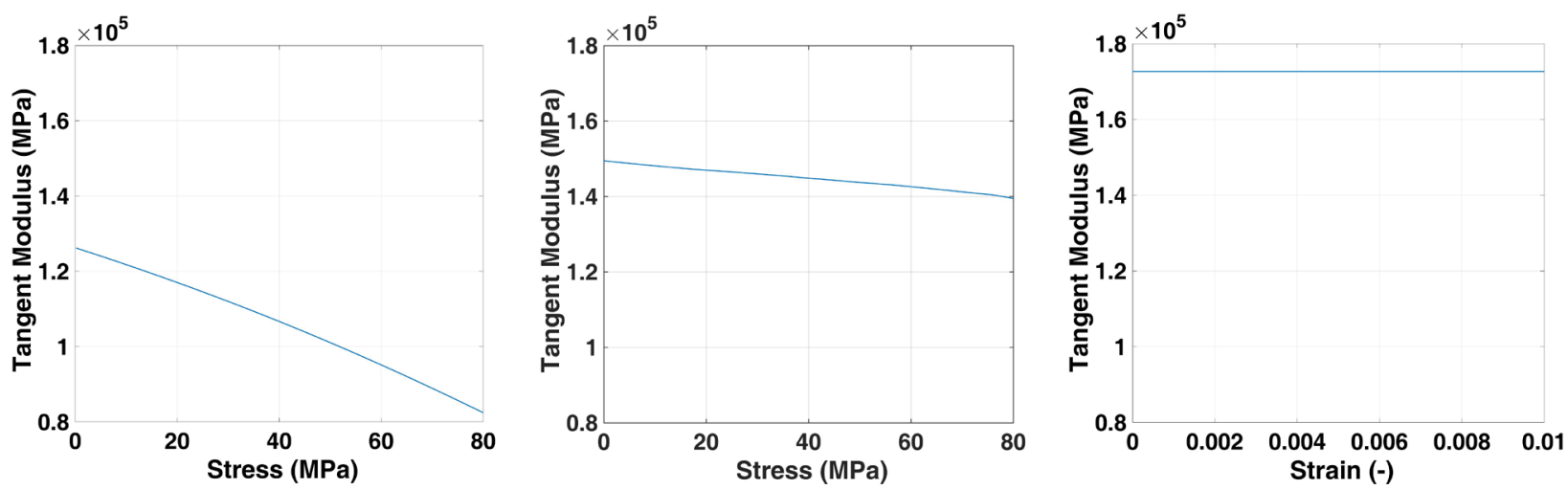

Figure 1. Tangent elastic module of LGI, compacted graphite iron (CGI) and SGI at different loads. 
In 1945, Hollomon [8] introduced an empirical equation describing the plastic part of the true stress-strain curve until necking. The Hollomon equation describes the plastic deformation using the strength coefficient, $k_{l}(\mathrm{MPa})$, and the strain hardening exponent, $n_{l}(-)$.

$$
\sigma=k_{1} \cdot\left(\varepsilon_{p}\right)^{n_{1}}
$$

Here $\sigma$ is the true stress, $\varepsilon_{p}$ the true plastic strain. The strain hardening exponent describes the ability of the material to resist further deformation, the higher the value the higher is the rate at which the material strain hardens. Using Eq.1 and considering the criterion, $\mathrm{d} \sigma / \mathrm{d} \varepsilon_{p}=0$, it can be shown that the strain hardening exponent equals the true plastic strain at necking, $\varepsilon_{p}=n_{l}$.

For some materials, the Hollomon equation does not describe the true stress-strain curve well at low plastic strains. By studying the deviation between the calculated and the measured true stressstrain curves a new term was added to the Hollomon expression by Ludwigson [9] to improve the fit, see Eq. 2, where $k_{2}$ and $n_{2}$ are material constants.

$$
\sigma_{\text {true }}=k_{1} \cdot \varepsilon_{p}^{n_{1}}+\exp ^{\left(k_{2}+n_{2} \cdot \varepsilon_{p}\right)}
$$

Figure 2 shows a typical tensile test curve and the application of the described models for SGI. The fit using the Hollomon expression can appear both over and under the experimental curve. As can be observed in Fig. 2, the Ludwigson approximation adds a correction to fit the experimental one better.

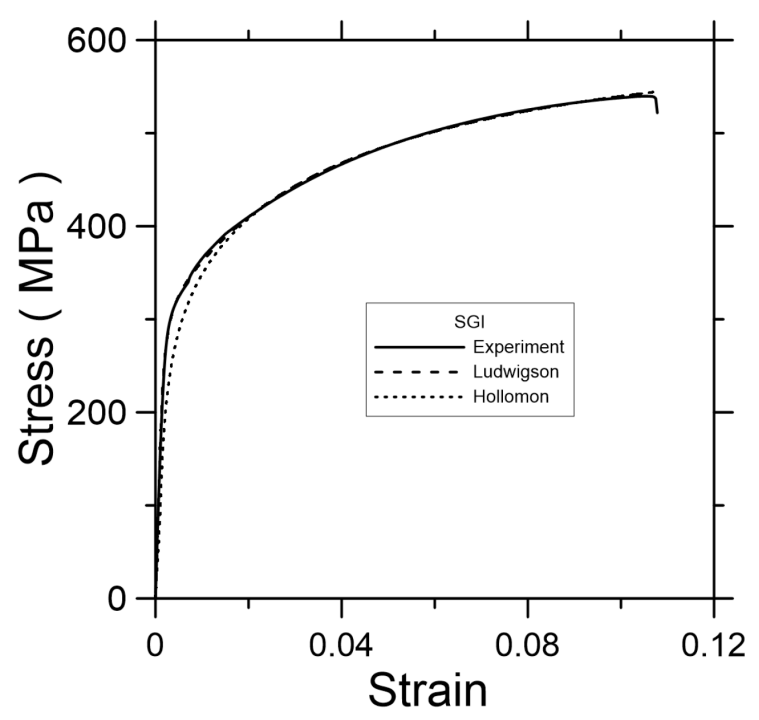

Figure 2. Typical tensile test curve for a SGI. The Hollomon and Ludwigson approximations have been applied by optimised parameters.

\section{Evaluation of Material Parameters from the Tensile Stress - Strain Curve}

The shape of the stress-strain curve is a result of how dislocations, vacancies and cracks are forming. Each combination of microstructure pattern and phase properties will give a curve which is a fingerprint of the material. By careful testing, data can be obtained to evaluate the plastic deformation behavior as well.

The engineering stress-strain curve from the tensile test machine must be re-calculated to give the true stress and true strain. Below, the transformation between engineering values and true stress and true strain are shown. The plastic deformation is calculated by subtracting the elastic part of the stress- strain curve.

The true elongation and stress are given by Eq. 3 and 5 and engineering (tech) values from true values in Eq. 4

$$
\varepsilon_{\text {true }}=\ln \left(1+\varepsilon_{\text {tech }}\right)
$$




$$
\begin{aligned}
& \varepsilon_{\text {tech }}=\exp \left(\varepsilon_{\text {true }}\right)-1 \\
& \sigma_{\text {true }}=\sigma_{\text {tech }}\left(1+\varepsilon_{\text {true }}\right) \\
& \varepsilon_{p}=\varepsilon_{\text {true }}-\frac{\sigma_{\text {true }}}{E} \\
& \varepsilon_{\text {true }}=\varepsilon_{p}+\frac{\sigma_{\text {true }}}{E}
\end{aligned}
$$

To get back to the engineering stress-strain curve, the following re-calculations have to be done.

$$
\begin{gathered}
\varepsilon_{\text {tech }}=\exp \left(\varepsilon_{p}+\frac{\sigma_{\text {true }}}{E}\right)-1 \\
\sigma_{\text {tech }}=\frac{\sigma_{\text {true }}}{\left(1+\varepsilon_{p}+\frac{\sigma_{\text {true }}}{E}\right)}
\end{gathered}
$$

The difference between the engineering curve (experiment) and the true curve (Ludwigson) is shown in Fig. 2.

\section{Methods to Fitting the Model Parameters to the Experimental Tensile Stress Curve}

After numerical treatment of the tensile curve, as explained in the section above, the curve is ready to be used for curve fitting.
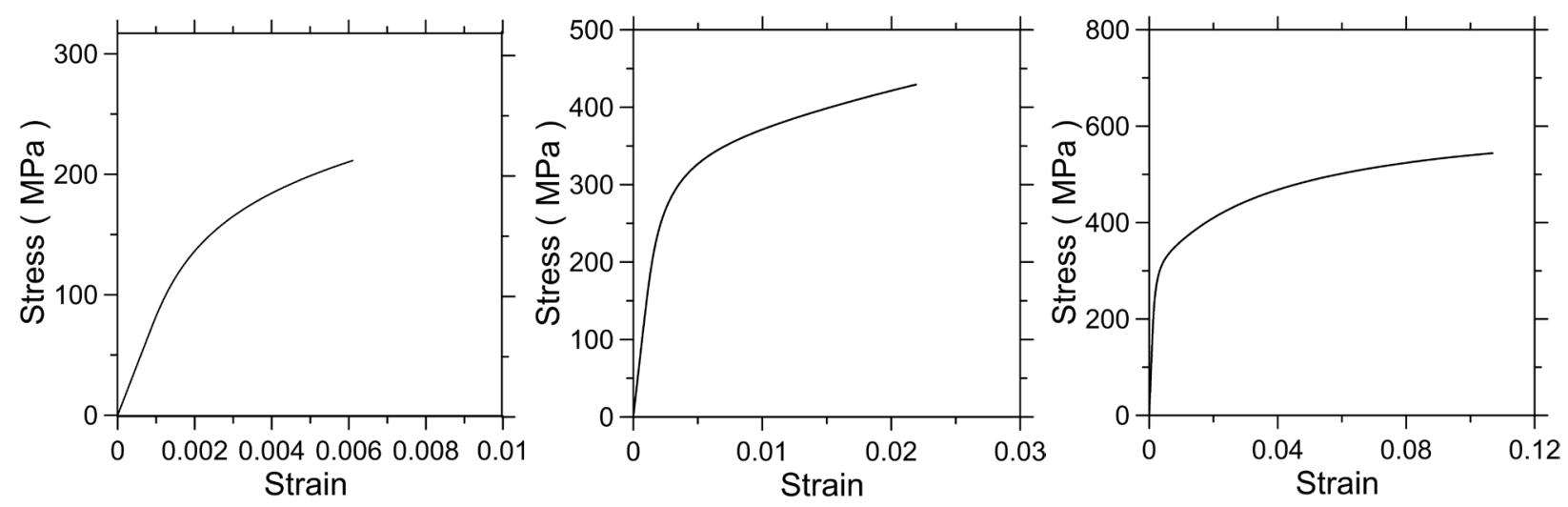

Figure 3. a-c Examples of tensile stress-strain curves, for LGI, CGI and SGI. The shape will be different depending on the microstructure and chemical composition. Observe that the graphs have different scales.

To capture the shapes of the curves, curve fitting must be used. Two well-known approximation models are described in the following.

\section{Hollomon model}

The Hollomon equation, Eq. 1, describes the plastic deformation behavior of a material. The total elastic and plastic deformation of the material is thus described by Young's modulus, $E$ (MPa), the strength coefficient [10], $k_{l}(\mathrm{MPa})$, and the strain hardening exponent, $n_{l}$.

The $k_{l}$ and $n_{l}$ parameters can be determined from a log-log plot of the true stress-true strain data or the constants can also be found numerically by optimization of the last part of the curve. The $n_{1^{-}}$ value corresponds to the slope of the curve and the $k_{l}$-value to the true stress at a true strain of unity. 


\section{Ludwigson model}

The Ludwigson equation contains an increased number of constants that needs to be determined. The parameters in the Ludwigson equation can be obtained by different logarithmic methods with good results.

Another way is to obtain the parameters to the equation by use of an optimization pattern search method. Pattern searching is a family of numerical optimization methods that do not require the gradient of the problem to be optimized. Hence pattern searching can be used on functions that are not continuous or differentiable. Such optimization methods are also known as direct-search, derivative-free, or black-box methods.

\section{Optimization Model of Hook - Jeeves}

Based on the true stress- true strain experimental curve, an approximation using either the Hollomon expression or the Ludwigson expression can be used to reverse engineer the parameters, $n_{1}, k_{1}, n_{2}$ and $k_{2}$ by the well-known Hooke and Jeeves pattern search optimization method [19, 20]. In fact, any choice of approximation can be used for this purpose. Considering the present study, the choice of approximation is the Ludwigson expression Eq. 2, i.e.,

$$
\sigma_{\text {sim }}=k_{1} \varepsilon_{p}^{n_{1}}+\exp \left(k_{2}+n_{2} \varepsilon_{p}\right)
$$

where now four different parameters need to be determined, namely $n_{1}, k_{1}, n_{2}$ and $k_{2}$. In order for the optimization algorithm to find an optimal solution, a so-called fitness functional needs to be created. Here, the $L_{2}$-norm of the error between the experimental true stress - true strain curve and the simulation, i.e. Eq. 10, is used according to

$$
f\left(n_{1}, k_{1}, n_{2}, k_{2}\right)=\sum_{i} \frac{1}{\xi_{i}}\left\|\sigma_{i}^{\text {sim }}-\sigma_{i}^{\exp }\right\|,
$$

where $\xi$ is used as a weight to choose more or less important parts of the curve fitting. It should be obvious that $\sigma^{\operatorname{sim}}$ and $\sigma_{i}^{\exp }$ corresponds to simulated and experimental stress, respectively.

The Hooke and Jeeves pattern search method will, based on an initial starting point $\boldsymbol{x}_{\text {init }}=$ $\left[n_{1}^{\text {init }}, k_{1}^{\text {init }}, n_{2}^{\text {init }}, k_{2}^{\text {init }}\right]$, vary each of the variables sequentially by a positive and negative variation such as, e.g., $\boldsymbol{x}_{+}^{n_{1}}=\left[n_{1}^{\text {init }}+\Delta n_{1}, k_{1}^{\text {init }}, n_{2}^{\text {init }}, k_{2}^{\text {init }}\right]$ and $x_{-}^{n_{1}}=\left[n_{1}^{\text {init }}-\Delta n_{1}, k_{1}^{\text {init }}, n_{2}^{\text {init }}, k_{2}^{\text {init }}\right]$ for the $n_{1}$ variable. In each variation, the fitness functional is evaluated which will yield three fitness function values and the point is updated according to the objective, i.e. in the present study the functional is minimized so that a lower value of the fitness is sought after. Once all variables have been varied, the size of the variation is reduced until the norm of the variation vector, i.e. $\Delta \boldsymbol{x}=$ $\left[\Delta n_{1}, \Delta k_{1}, \Delta n_{2}, \Delta k_{2}\right]$, is within a predefined tolerance. Thus, it is of importance that the initial variation is set large enough so that a local minima or global minimum can be obtained by the optimization algorithm.

In the case of SGI, the Ludwigson approximation model can be used, but in some cases the Hollomon model describes the stress-strain curve well enough.

\section{Giving the Fitting Parameters a Physical Meaning}

The optimization can fit most curves but it can be very difficult to relate the obtained parameters to the microstructure and the mechanical properties. Typical stress-strain curves are shown in Fig 3. The Hollomon approximation model is well-known for many homogenous materials and it is easier to relate microstructure to the deformation behavior of the material for a tensile test.

In order to relate microstructure to mechanical properties and use the tensile test curve as a response at the properties, the following schematic is proposed. The results should be comparable if they are made in different laboratories.

1. Make a log-log fitting of the curve to get the Hollomon parameters or make an optimization of the Hollomon equation for the last part of the tensile curve. 
2. Take the parameters obtained in the first step and perform an optimization of the whole curve by adding the parameters of the Ludwigson correction to the approximation.
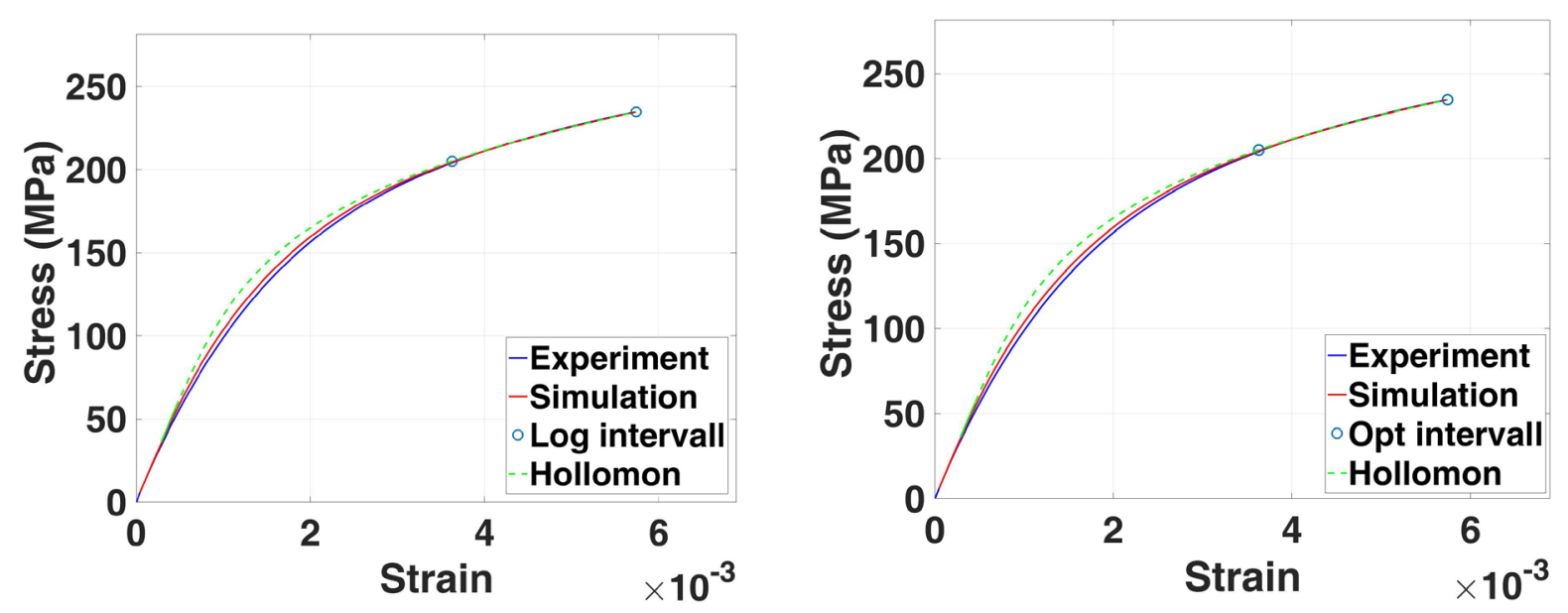

Figure 4. Lamellar graphite iron tensile test curve (left) with a double logarithm of the last part of the plastic curve, solid line corresponds to the experiment and the dashed line the fitted result. In the right graph, using the Hollomon parameters in the optimization of the whole curve and using the

Ludwigson correction.

\section{Understanding Deformation Behavior Using the Tensile Test Curve}

The tensile stress-strain curve reflects the behavior of the material under stress. Even if the load is low we can expect that some parts of the microstructure will be deformed and result in a decohesion between the graphite and the matrix phase [11]. Using acoustic emission [12], the formation of cracks can be detected and the more cracks the louder is the sound emitted and more events can be observed. The second part which we normally refer to as plastic deformation in a cast iron represent a mixture of crack formation and growth of the cracks as well as formation of dislocations, commonly called deformation hardening. The properties of a cast iron depend on the microstructure [13-16,18] and the properties will be dependent on the local microstructure. By connecting the local tensile stress-strain curves in a casting component with simulations of the local microstructure we can obtain better predictions of a component's behavior during loading, meaning that more realistic distribution of material properties data can be input in to finite element analyses [17].

\section{Summary}

A method has been demonstrated on how to characterize a tensile stress-strain curve into four parameters. The mathematical solution is based on the Hooke and Jeeves pattern search method. To compare the plastic behavior with the Hollomon equation, the optimization is done in two steps, first the parameters in the Hollomon expression are determined and then an optimization is performed to determine the Ludwigson correction parameters. This mathematical description is necessary in order to understand the relationship between microstructure and mechanical properties, furthermore for the next generation of simulation software where local microstructures will influence the mechanical behavior of a component with inhomogeneous properties. 


\section{References}

[1] J. Olofsson, Simulation of Microstructure-based Mechanical Behavior of Cast Components, Ph.D. thesis, (Jönköping University, School of Engineering, 2014).

[2] T. Sjögren, I.L. Svensson, Studying Elastic Deformation Behavior of Cast Irons by Acoustic Emission, Int. J Cast Met Res. 18 (2005) 249-256

[3] P. Mazilu, G. Ondracek, On the Effective Young's Modulus of Elasticity for Porous Materials, Part I: The General Model Equation, in: Herrmann K., Olesiak Z. (eds.), Thermal Effects in Fracture of Multiphase Materials. Proc. Euromech. Colloquium 255. Springer Verlag Heidelberg Tokyo New York pp. 214-230

[4] A.R., Boccaccini, Young's Modulus of Cast-Iron as a Function of Volume Content, Shape and Orientation of Graphite inclusions, Z. Metallkd. 88 (1997) 23-26

[5] F. Wilberfors, I.L. Svensson, The Effect of Nitrogen and Inoculation on The Tensile Properties and Microstructure of Cast Iron with Lamellar Graphite, Key Eng. Mater. 457 (2011) 114-119

[6] SS-EN ISO 6892-1:2009(E): Metallic materials - Tensile testing - Part 1: Method of test at room temperature, B. European Committee for Standardization.

[7] J. R. Davis editor \& Associates, Tensile testing $2^{\text {nd }}$ edition, ASM International, ISBN 0-87170 $\mathrm{X}(2004)$

[8] H. Hollomon, Trans. AIME 162, (1945), 268

[9] D. C. Ludwigson, Modified stress-strain relation for FCC metals and alloys, Metal. Trans. 2, (1971) 2825-2828.

[10] T. Sjögren, I.L. Svensson, Modelling the effect of graphite morphology on the modulus of elasticity in cast irons, Int. J. Cast Metal. Res. 17(5) (2004) 271-279.

[11] J. Olofsson, I.L. Svensson, Casting and stress-strain simulations of a cast ductile iron component using microstructure based mechanical behavior, IOP Conf. Series: Materials Science and Engineering 33 (2012) 012051, Conf. on Modeling of Casting, Welding and Advanced Solidification Processes (MCWASP XIII) June 17-22, 2012 in Schladming, Austria

[12] T. Sjögren, Studying elastic deformation behaviour of cast irons by acoustic emission, Int. J. Cast Metal Res. 18(4) (2005) 249-256.

[13] I.L. Svensson, T. Sjögren, On modeling and simulation of mechanical properties of cast irons with different morphologies of graphite, Int. J. Metalcast. 3 (4) (2009) 67-77

[14] I.L. Svensson, J. Olofsson, On microstructure-based mechanical behaviour of a ductile iron component, $10^{\text {th }}$ International Symposium on the Science and Processing of Cast Iron - SPCI10 2014, Mar det plata, Argentina.

[15] J. Olofsson, K. Salomonsson, I.L. Svensson, Modelling and simulations of ductile iron solidification-induced variations in mechanical behaviour on component and microstructural level, IOP Conf. Ser.-Mat. Sci. 84 (2015), 012026

[16] K. Kasvayee, K. Salomonsson, E. Ghassemali, Anders E.W. Jarfors, Microstructural strain distribution in ductile iron; comparison between finite element simulation and digital image correlation measurements, Mat. Sci. Eng. A - Struct. 655 (2016) 27-35.

[17] J. Olofsson, I.L. Svensson, Incorporating predicted local mechanical behaviour of cast components into finite element simulations, Materials \& Design 34 (2012) 494-500.

[18] H. Fredriksson, I.L. Svensson, Computer Simulation of the Structure Formed During Solidification of Cast Iron, in: H. Fredriksson, M. Hillert (eds.) The Physical Metallurgy of Cast Iron: proceedings of the Third International Symposium on the Physical Metallurgy of Cast Iron, Stockholm, Sweden, August 29-31, 1984, New York: North-Holland, 1985, pp. 273-284

[19] R. Hooke, T.A Jeeves, "Direct search" solution of numerical and statistical problems, J. Assoc. Comput. Mach. 8 (2) (1961) 212-229.

[20] W.C. Davidon, Variable metric method for minimization, SIAM J. Optimiz. 1 (1) (1991) 1-17. 